\title{
FEM Simulation of the Martensitic Tranformation in NiTi Alloys
}

\author{
S. Zhang, H. Braasch* and P.G. McCormick
}

Department of Mechanical and Materials Engineering, University of Western Australia, Nedlands, WA 6907, Australia

* DYWIDAG, Landstrasse 1, 81902 München, Germany

\begin{abstract}
Numerous studies have demonstrated the influence of elastic and plastic strain energies on the martensitic transformation in shape memory alloys. In recent years the Finite Element Method (FEM) has been applied to a wide range of elastic and plastic problems, however, few FEM analyses of shape memory phenomena have been reported. In this paper the FEM method has been used to model the martensitic transformation. The model assumes a two variant shear transformation in a two dimensional grain. Plastic deformation accompanying the transformation is accounted for using the Estrin-Mecking constitutive model. The spatial distribution of elastic and plastic strain energies developed during the transformation is shown to determine variant structure. Calculations of the effect of applied stress on variant structure and the effect of stress and repeated thermal cycling on the transformation temperatures are presented.
\end{abstract}

\section{INTRODUCTION}

In recent years a number of models for shape memory alloys have been proposed based on Landau's theory of phase transition, statistics and continuum mechanics and thermodynamics [1-6]. However, few Finite Element Method (FEM) analyses of shape memory phenomena have been reported $[7,8] . \mathrm{Xu}[7,8]$ reported a model which describes the martensitic transformation process with or without an applied stress based on the linear elastic model developed by Khachaturyan [9]. In this paper the FEM method has been developed to model the martensitic transformation in NiTi. In order to simulate a simple case, it is assumed that there are only two types of martensitic variants and the transformation strain is pure shear in a two dimensional grain. Using the Estrin-Mecking constitutive model, calculation of plastic deformation accompanying the transformation is carried out. The spatial distribution of elastic and plastic strain energies developed during the transformation is shown to determine variant structure and the transformation process and temperature.

\section{FINITE ELEMENT METHOD MODEL}

\subsection{Finite element model}

It is assumed that the transformation occurs in a grain modelled by an ( $\mathrm{n} \times \mathrm{n}$ ) square grid in twodimensional space surrounded by a boundary consisting of an untransformed layer of material elastically coupled to the outer rigid boundary. Each square represents the minimum unit that can transform and this unit is called the elementary cell of the transformation.

Each square in the grain is an isoparametric and quadratic finite element of eight nodes. If the relationship between stress $\sigma$ and strain $\varepsilon$ is written in the form

$$
\sigma=\mathbf{D}\left(\varepsilon-\varepsilon_{0}\right)+\sigma_{0}
$$


where $D$ is an elastic matrix, $\sigma_{0}$ and $\varepsilon_{0}$ are the initial stress and strain respectively, according to elastic FEM theory [10], we have a system of ordinary equations

where $\quad \mathbf{K}=\int_{\mathbb{V}} \mathbf{B}^{\mathrm{T}} \mathbf{D B d V}, \quad$ and $\mathbf{f}=-\int_{V}^{\mathbf{K a}=\mathbf{r}-\mathbf{f}} \mathbf{N}^{\mathrm{T}} \mathbf{b d V}-\int_{V} \mathbf{N}^{\mathrm{T}} \mathrm{tdV}-\int_{V} \mathbf{B}^{\mathrm{T}} \mathbf{D} \boldsymbol{\varepsilon}_{0} \mathrm{dV}-\int_{V} \mathbf{B}^{\mathrm{T}} \sigma_{0} \mathrm{dV}$

where $t$ is the boundary loads $\mathrm{V}$ the volume of the whole body, $\mathbf{B}$ is the differential operator matrix and $\mathbf{N}$ is a shape function required from the FEM. The $\mathbf{a}$ and $\mathbf{r}$ are the nodal displacements and external forces applied on the nodes respectively.

For a non-linear plastic FEM problem, we have

$$
\mathrm{d} \sigma=\mathbf{D}_{\mathrm{cp}} \mathrm{d} \varepsilon
$$

where the elasto-plastic matrix $\mathbf{D}_{\mathrm{ep}}$ is dependent on the yield criterion, the hardening function and the elastic matrix D. Solving eqs. (1) - (3) using the Newton-Raphson method for iteration, the strain and stress increment $\Delta \varepsilon$ and $\Delta \sigma$ for each equilibrium solution and the final strain and stress $\varepsilon$ and $\sigma$ can be obtained.

\subsection{The transformation strain and the mechanical energy}

For simplicity, a two variant model is assumed. Since there is a very small volume change of the martensitic transformation in shape memory alloys, only the shear components of the transformation are taken into account. So the initial strains in eq. (1) contain the engineering shear strain component $\gamma_{1}=\gamma_{0}$ for variant 1 , and $\gamma_{2}=-\gamma_{0}$ for variant 2 .

With initial stress $\sigma_{0}$, the transformation strain $\varepsilon_{0}$ occurs in a grain of volume $V$. In order to calculate the system energy change induced by the transformation, there are three steps in this elastic model [9].

(1) Cut the region $v_{0}$ in which the transformation will happen from the matrix (the grain) and remove it. At the moment, the transformation occurs under initial stress $\sigma_{0}$ and is boundary-free, no elastic energy is produced but the work due to the initial stress $\sigma_{0}$ and the transformation strain $\varepsilon_{0}$ exists and is equal to

$$
\mathrm{w}_{0}=-\int_{\mathrm{V}_{0}}^{\mathrm{r}} \boldsymbol{\sigma}_{0} \mathrm{dV}
$$

(2) In order to put the transformed region into original position, apply a force on the region and restore its original shape, then put it back into the matrix. The increase of elastic energy stored in the transformed region is obtained in this step and can be written as

$$
\Delta \mathrm{E}_{\text {store }}=\int_{\mathrm{V}_{0}}\left(\frac{1}{2} \boldsymbol{\varepsilon}_{0}^{\mathrm{T}} \mathbf{D} \varepsilon_{0}-\varepsilon_{0}^{\mathrm{T}} \sigma_{0}\right) \mathrm{dV}
$$

It should be noted that there is not any elastic energy change in the matrix as yet.

(3) Remove the force applied in step (2) from the region. The relaxation of the transformed region gives the elastic strain energy change in both the transformed region and the matrix

$$
\Delta \mathrm{E}_{\text {relax }}=\int\left(\frac{1}{2} \varepsilon^{\mathrm{T}} \mathbf{D} \varepsilon+\varepsilon^{\mathrm{T}} \sigma_{0}\right) \mathrm{dV}-\int_{v_{0}}^{\mathrm{T}} \mathbf{D} \varepsilon_{0} \mathrm{dV}
$$

The combination of equations (4), (5) and (6) gives the total elastic and transformation energies produced by the transformation.

In all shape memory alloys plastic deformation invariably accompanies the martensitic transformation [11-15]. In this model, the Estrin-Mecking constitutive model [16-17] for plastic deformation is used. Using the Estrin-Mecking plastic model gives the relationship between stress and the plastic strain. When the plastic deformation is considered, the mechanical energy change due to the transformation can be expressed in the form [18]

$$
\Delta \mathrm{E}_{\text {total }}=\mathrm{w}_{0}+\Delta \mathrm{E}_{\text {ep }}=-\int_{\mathrm{N}_{0}} \varepsilon_{0}^{\mathrm{T}} \sigma_{0} \mathrm{dV}+\int_{\mathrm{V}}\left[(\Delta \varepsilon)^{\mathrm{T}} \sigma-\frac{1}{2}\left((\Delta \varepsilon)^{\mathrm{T}} \Delta \sigma\right)\right] \mathrm{dV}
$$


where $\Delta \mathrm{E}_{\mathrm{ep}}$ is the elastic and plastic energy produced by the transformation, $\Delta \varepsilon, \Delta \sigma$ and $\sigma$ represent the strain and stress increment as well as the stress of each step for equilibrium respectively. The Von Mises yield criterion for plastic deformation is employed.

\subsection{The free energy and the transformation path}

The total Gibbs free energy change $\Delta \mathrm{G}$ due to the transformation can be written as

$$
\Delta \mathrm{G}=\Delta \mathrm{G}_{\mathrm{c}}+\Delta \mathrm{E}_{\text {total }}
$$

where the chemical free energy, $\Delta \mathrm{G}_{\mathrm{c}}$, is assumed to be proportional to temperature $T$

$$
\Delta \mathrm{G}_{\mathrm{c}}=\mathrm{K}\left(\mathrm{T}_{\mathrm{o}}-\mathrm{T}\right)
$$

where $T_{0}$ is the equilibrium temperature and $K$ is equal to entropy change $\Delta S_{c}$. Therefore, for an elastic matrix, the total free energy change due to the transformation is given by eqs. (4), (5), (6) and (9). For the plastic case, the use of eqs. (7), (8) and (9) yields the total free energy change. It is assumed that the irreversible energy of the transformation is produced by plastic deformation only.

In a grain with an ( $\mathrm{x} \times \mathrm{n}$ ) square grid, there are $\mathrm{n}$ plates, each plate contains $\mathrm{n}$ squares and is parallel to the one edge of the boundary. If an elementary plate is the smallest unit that can transform, the transformation proceeds plate by plate. At the beginning, there are $2 \mathrm{n}$ choices for the first plate to transform since two kinds of martensitic variants are assumed in this model. After calculating the Gibbs free energy change for each position and each variant respectively, the position and variant with the minium free energy change will be the first plate to transform. The transformation temperature for this variant is calculated from eq. (9) with $\Delta \mathrm{G}=0$. When the first plate is transformed, the same procedure is repeated for the second plate but the choices that can be taken will be reduced by 2 , that is, there are 2 (n1) remaining choices for the second plate. For the other plates to transform, this procedure is followed until the whole grain is transformed. The reverse transformation is treated in the same way.

\section{RESULTS AND DISCUSSION}

In this section the results of a simulation of the forward and reverse martensitic transformation during a thermal cycle under constant stress are given. The properties of NiTi shape memory alloys and other parameters used in the simulation are shown in table 1.

Using the minimum energy method described above, the first martensite plate transformed as variant 1 at the top of the grain, variant 1 being favoured by the applied stress. The elastic and plastic strain fields accompanying the formation of the variant were concentrated in the boundary adjacent the ends of the variant. The second martensite plate was also variant 1 and was located at the bottom of the grain. The elastic, plastic and total strain fields produced by the second plate were similar to the strain fields produced by the first plate. Following the minimum energy procedure the entire grain was transformed plate by plate to martensite.

Fig. 1 shows the spatial distribution of the elastic and plastic strain energies after the forward transformation. It is seen that the elastic and plastic strain fields are located mainly in the untransformed boundary at the end of the plates. The elastic strain energy opposes the transformation and is limited by the occurrence of plastic deformation. With an applied stress of $250 \mathrm{MPa}$ all of the variants were of type 1. It was found that stresses greater than $200 \mathrm{MPa}$ were required to obtain $100 \%$ of variant 1 . With lower stresses increasing self accommodation occurred as the stress was lowered.

The elastic and plastic strain energies after a complete forward and reverse transformation cycle are shown in Fig. 2. The strain fields remain concentrated in the boundary region. It is noted that the residual elastic strain energy is the result of the plastic strain induced by the transformation, as in the absence of any plastic strain the transformation must be fully reversible. The residual elastic strain fields occur in the direction to assist the forward transformation of variant 1 under zero stress, a requirement for two-way shape memory behaviour. It is seen that little change in the plastic energy occurred during the reverse cycle. 
In Fig. 3 the fraction of transformed martensite is plotted as a function of the temperature. The curve exhibits the experimentally observed features of transformation hysteresis and a temperature interval between the transformation start and finish. The hysteresis clearly results from the (irreversible) plastic deformation caused by the transformation. The sequence of transforming plates for the reverse transformation was exactly opposite to that for the forward transformation, as expected for a thermoelastic transformation.

The effect of repeated thermal cycling on the hysteresis is shown in Fig. 4. The hysteresis, $\Delta T_{h}$, decreases progressively with increasing number of cycles due to the effect of strain hardening, which is accounted for in the simulation by the Estrin-Mecking model. Similar behaviour is observed experimentally [15], however, $\Delta T_{h}$, normally does not decrease to zero as shown in Fig. 4 . A non-zero value of $\Delta T_{h}$ after a large number of cycles may be modelled by inclusion of a constant energy term which is associated with the frictional and other irreversible transformation energy losses.

The effect of the applied stress on the transformation temperature has also been modelled. In Fig. 5 the applied stress is plotted as a function of the temperature required for the first martensitic variant to form. Also shown in Fig. 5 is the stress-temperature curve obtained using an elastic model, ie. no plastic deformation. It is seen that the two curves diverge at high stresses.

The temperature dependence of the transformation stress is usually modelled by the ClassiusClapeyron like relation

$$
\mathrm{d} \sigma / \mathrm{dT}=-\Delta \mathrm{H} / \mathrm{T}_{0} \varepsilon_{0}
$$

The slope of the elastic curve is identical with that calculated from eq. (10) using the parameters in Table 1. Eq. (10) does not take into account any stress dependence of the plastic energy as would be expected for strain hardening. To take strain hardening into account, eq. (10) may be rewritten as [19]:

$$
\mathrm{d} \sigma / \mathrm{dT}=-\Delta \mathrm{H} /\left(\mathrm{T}_{0}\left(\varepsilon_{0}-\partial\left(\Delta \mathrm{E}_{\mathrm{p}}\right) / \partial \sigma\right)\right)
$$

where $\Delta \mathrm{E}_{\mathrm{p}}$ is the plastic energy. The second term in the denominator of eq. (10) increases with increasing stress and is of opposite sign to $\varepsilon_{0}$, thus explaining why the two curves diverge.

Table 1 Input data for the calculation in FEM model

\begin{tabular}{|c|c|c|c|c|c|}
\hline Properties & $\begin{array}{c}\text { Young's } \\
\text { modulus } \\
(\mathrm{MPa})\end{array}$ & $\begin{array}{c}\text { Poisson's } \\
\text { ratio }\end{array}$ & $\begin{array}{c}\text { Yield } \\
\text { Strength } \\
(\mathrm{MPa})\end{array}$ & $\begin{array}{c}\text { Hardening } \\
\text { Constant } \\
\mathrm{C}_{1} \text { and } \mathrm{C}_{2}\end{array}$ & $\begin{array}{c}\text { Equilibrium } \\
\text { temperature } \\
\mathrm{T}_{0}(\mathrm{~K})\end{array}$ \\
\hline NiTi alloys & 60,000 & $1 / 3$ & 600 & 25 and 12 & 320 \\
\hline Properties & $\begin{array}{c}\text { Enthalpy } \\
\text { change } \Delta \mathrm{H}_{0} \\
(\mathrm{~J} / \mathrm{g})\end{array}$ & $\begin{array}{c}\text { Transformation } \\
\text { Strain } \gamma_{0}\end{array}$ & $\begin{array}{c}\text { Spring } \\
\text { constant } \\
(\mathrm{N} / \mathrm{mm})\end{array}$ & $\begin{array}{c}\text { Size of Grain } \\
(\mathrm{mm} \times \mathrm{mm})\end{array}$ & \\
\hline NiTi alloys & -20 & 0.128 & 300 & $0.1 \times 0.1$ & \\
\hline
\end{tabular}

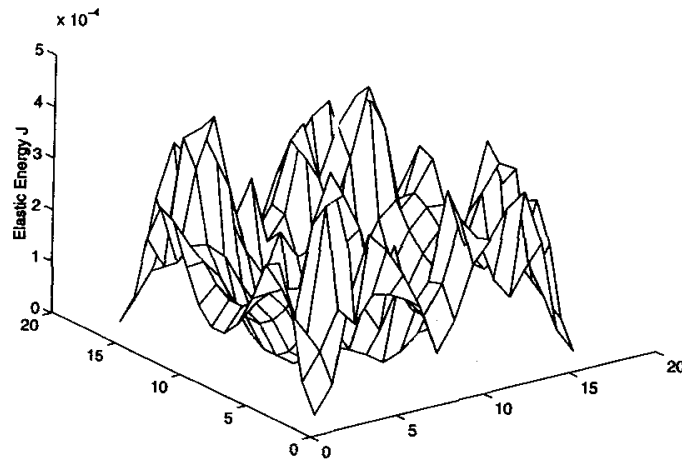

(a)

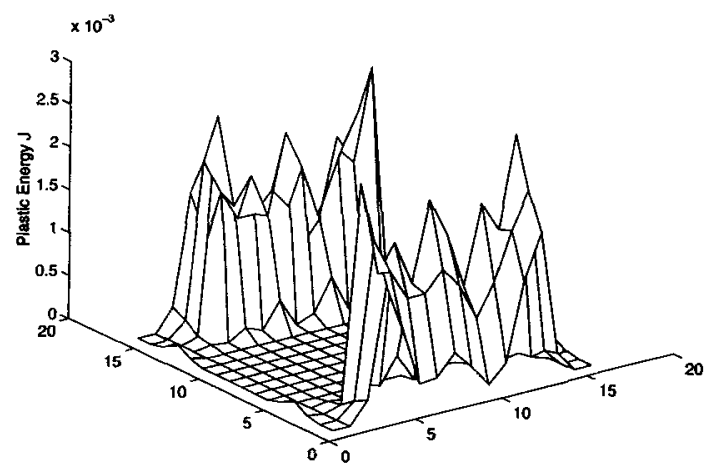

(b)

Fig.1 Distribution of energy after the forward transformation (a) Elastic, (b) Plastic (external shear stress: 250 MPa) 


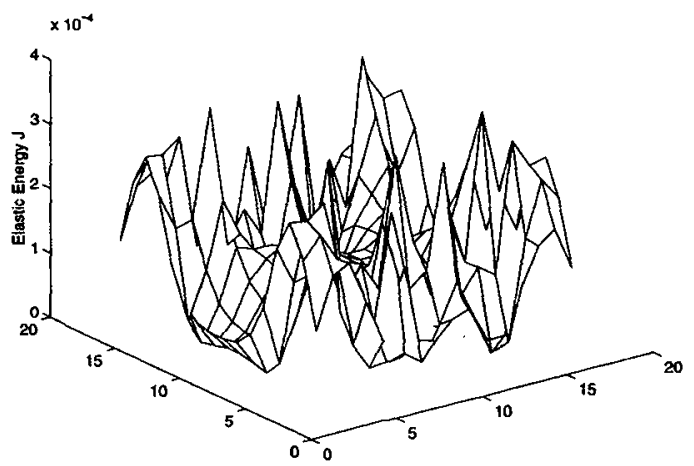

(a)

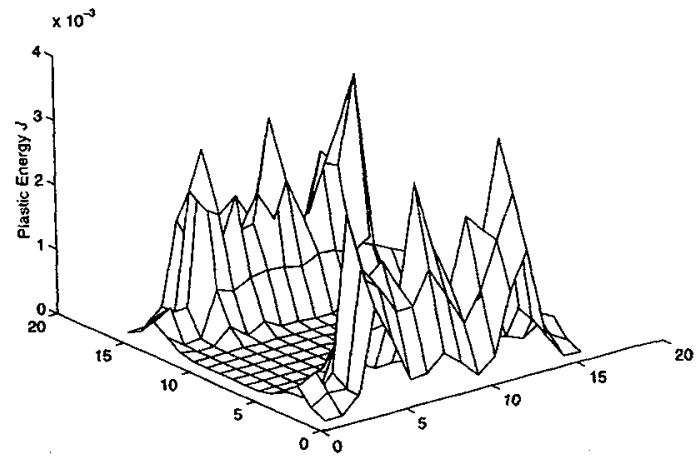

(b)

Fig.2 Distribution of energy after the reverse transformation (a) Elastic, (b) Plastic (external shear stress: $250 \mathrm{MPa}$ )

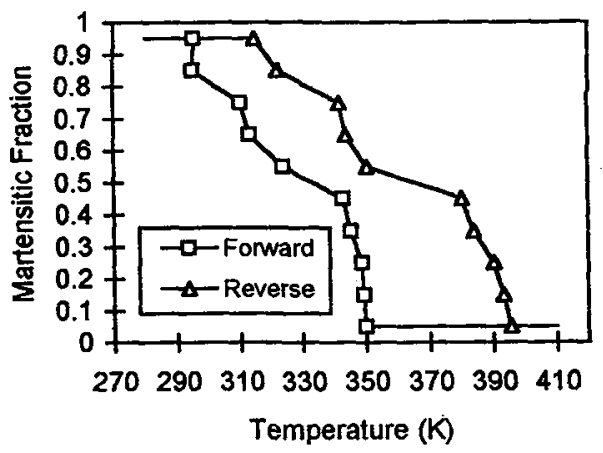

Fig. 3 Curves of temperature and martensitic fraction (applied shear stress $300 \mathrm{MPa}$ )

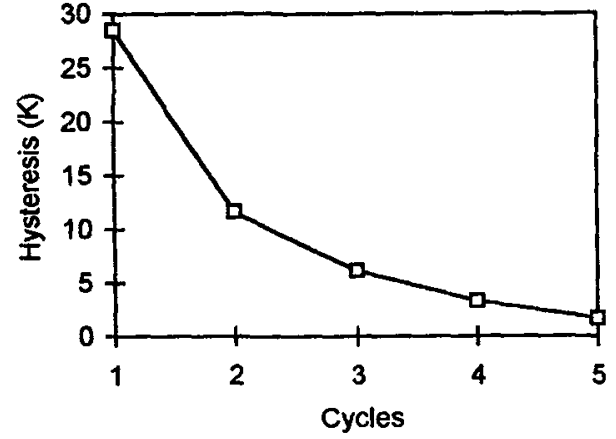

Fig.4 Effect of thermal cycling with load on the hysteresis (applied shear stress $300 \mathrm{MPa}$ )

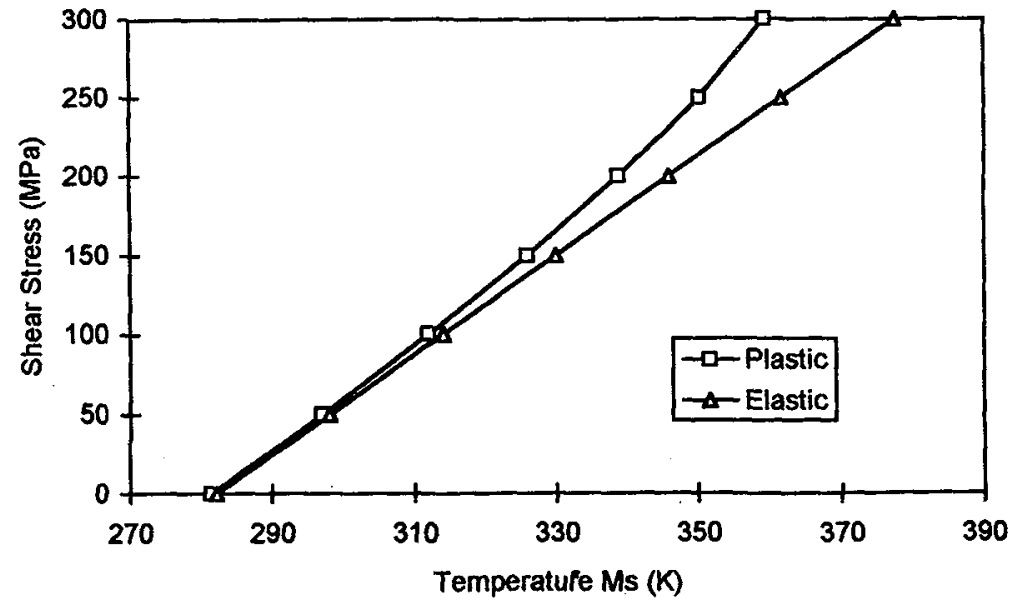

Fig.5 Effect of applied stress on Ms 


\section{CONCLUSIONS}

This paper illustrates a few of the insights that can be obtained from FEM modelling of the martensitic transformation and shape memory behaviour. Although the results of the FEM model are in qualitative agreement with experiment, it is clear that a number of refinements are necessary to more closely model experimentally observed behaviour. In particular, a much larger array needs to be used. With the present work, the available computing facilities limited the analysis to only a $11 \times 11$ array and two variants. It is well known that there are 24 variants in shape memory alloys and the combination of different deformations produced by the different variants gives the macroscopic deformation behaviour of these alloys. Account needs to be taken of the differing elastic and plastic properties of the parent and martensitic phases. The boundary conditions also need to be examined in detail as they can significantly influence the results of the simulation. Nonetheless, as well as being in qualitative agreement with experiment, the results obtained here illustrate the interplay of the elastic and plastic response to the martensitic transformation.

\section{References}

[1] F. Falk, Acta Metall., 28 (1980) 1773-1780.

[2] I. Muller and K. Wilmanski, II Nuovo Cimento, 57B (1980) 283-318.

[3] Y. Sato, K. Tanaka and S. Kobayashi, Trans. Japan Soc. Aero. Space Sci. 28 (1985) 150-160.

[4] K. Tanaka, S. Kobayashi and Y. Sato, Inter. J. Plasticity, 2 (1986) 59-72.

[5] E. Patoor, A. Eberhardt and M. Berveiller, Arch. Mech., 40 (1988) 775-794.

[6] C. Liang and C. A. Rogers, J. Intell. Mater. Syst. And Struc., 1 (1990) 207-234.

[7] P. Xu and J. W. Morris, Metall. Trans. A, 23A (1992), 2999-3012.

[8] P. Xu and J. W. Morris, Metall. Trans. A, 24A (1993), 1281-1294.

[9] A. G. Khachaturyan, Theory of Structure Transformations in Solids, (Wiley, New York, 1983) 198277.

[10] O. C. Zienkiewicz, The Finite Element Method, (McGraw-Hill Book Company, The Third edition, 1977) 20-40.

[11] T. Saburi, T. Tatsumi and S. Nenno, Journal De Physique, C4 (1982) 261-266.

[12] T. Saburi, M. Yoshida and S. Nenno, Scripta Metall., 18 (1984) 363-366.

[13] S. Miyazaki and K. Otsuka, Metall. Trans., 17A (1986) 53-63.

[14] G. B. Stachowiak and P. G. McCormick, Acta Metall., 36 (1988) 291-297.

[15] P. G. McCormick and Y. Liu, Acta Metall. Mater., 42 (1994) 2407-2413.

[16] Y. Estrin and H. Mecking, Acta Metall., 32 (1984) 57-70.

[17] Y. Estrin and H. Mecking, Int. J. Plasticity, 1 (1985) 73-85.

[18] S. Zhang, Modelling of Shape Memory Alloys, Ph. D Thesis, Univ. of Western Australia (1997).

[19] S. Zhang and P. G. McCormick, [to be published]" 